\title{
Case Report of a Rare Adrenocortical Oncocytoma Suspected to be an Adrenal Carcinoma
}

\author{
Inês Isabel Ferreira Barros, ${ }^{1}$ Fernando Manso, ${ }^{2}$ Margarida Teixeira ${ }^{3}$ and Maria Ramires Silva Lopes Pereira ${ }^{1}$
}

1. Department of Endocrinology, Braga Public Hospital, Braga, Portugal; 2. Department of General Surgery,

Braga Public Hospital Braga, Portugal; 3. Department of Anatomic Pathology, Braga Public Hospital, Braga, Portugal

DOI: https://doi.org/10.17925/EE.2021.17.1.71

A n adrenal oncocytic neoplasm is an extremely rare tumour arising from the adrenal gland and it should be considered in the differential diagnosis of an adrenal incidentaloma, since it is frequently non-functioning. The suspicion for malignancy is high when an adrenal incidentaloma is $>4 \mathrm{~cm}$ in size; however, adrenal oncocytomas are large, measuring an average of $8 \mathrm{~cm}$, are round and encapsulated, and normally have a benign behaviour. We present a case of a 55-year-old male patient with dyslipidaemia, medicated with simvastatin. Upon complaints of abdominal pain, the general physician asked for an abdominal ultrasound that revealed an adrenal lesion, further characterized with a computed tomography scan, which showed an adrenal lesion measuring $49 \times 64 \times 56 \mathrm{~mm}$ and a calcification focus. The patient was referred to the general surgery and endocrinology department. The analytical study was negative for pheochromocytoma or Cushing's syndrome, which allowed surgery to be conducted, as is recommended. The aim of this case report is to contribute to the knowledge on adrenal oncocytomas, since there is scarce information based on singular experiences.

\section{Keywords}

Adrenocortical oncocytoma, adrenocortical carcinoma, adrenal incidentaloma, oncocytoma

Disclosures: Inês Ferreira Barros, Fernando Manso, Margarida Teixeira and Maria Ramires Silva Lopes Pereira have no financial or non-financial relationships or activities to declare in relation to this article.

Review process: Double-blind peer review. Compliance with ethics: Informed consent was received from the patient included in this case report.

Authorship: The named authors meet the International Committee of Medical Journal Editors (ICMJE) criteria for authorship of this manuscript, take responsibility for the integrity of the work as a whole, and have given final approval for the version to be published. Access: This article is freely accessible at touchENDOCRINOLOGY.com (c) Touch Medical Media 2020. Received: 13 September 2020

Accepted: 28 October 2020

Published Online: 22 March 2021

Citation: touchREVIEWS in Endocrinology. 2021;17(1):71-4

Corresponding Author: Inês Isabel Ferreira Barros, Sete Fontes, São Victor, 4710-243, Braga, Portugal.

E: barros.ines@gmail.com; ines.barros@hb.min-saude.pt

Support: No funding was received in the publication of this article.
Oncocytomas are epithelial tumours composed of cells with eosinophilic, mitochondria-rich cytoplasm. ${ }^{1}$ They can occur in various organs, such as the kidneys, thyroid, pituitary gland, salivary glands and parathyroid glands. Rarely, it has been reported in the respiratory and gastrointestinal tracts. ${ }^{2-4}$ Adrenal oncocytomas are extremely rare, with nearly 200 published cases since Kakimoto et al. reported the first case in $1986.5,6$

The detection of adrenal oncocytomas is mostly incidental since they are frequently non-functional; however, biochemical analyses are recommended to exclude functional status. ${ }^{7}$ Imaging is not a reliable method of diagnosis for oncocytomas as they can display features often present in other tumours, such as carcinoma; histological analysis is required for an accurate diagnosis. since oncocytomas are benign lesions associated to favourable prognosis, the diagnosis has implications for follow up. Despite the impermissible indication for surgery, there are scarce data concerning follow-up strategies. ${ }^{7.8}$

\section{Case presentation}

We present a 55-year-old male patient with dyslipidaemia and important ethylic habits. He is a former smoker with a medical history of sacral-coccygeal cyst, traumatic amputation of two left-hand fingers in 2000, a peroneal fracture in 2014 and is medicated with simvastatin 20 mg daily. In 2017, the patient complained to his general physician of abdominal pain. Abdominal ultrasound revealed a right adrenal lesion, further characterized with a computed tomography (CT) scan that showed a nodular heterogeneous lesion measuring $49 \times 64 \times 56 \mathrm{~mm}$ with a calcification focus in the right adrenal gland. In this context, the patient was referred to the general surgery and endocrinology department in July 2017.

During the endocrinology appointment for an adrenal incidentaloma, we requested a hormonal study to evaluate the possibility of a functional mass and a renal CT scan. Since the patient did not have hypertension, we focused on pheochromocytoma and Cushing's syndrome exclusion. The results of this study (in May 2018) were: $1 \mathrm{mg}$ overnight dexamethasone suppression test of 2.9 $\mu \mathrm{g} / \mathrm{dL}$ (normal range $<1.8 \mu \mathrm{g} / \mathrm{dL}$ ), adrenocorticotropic hormone (ACTH) of $22 \mathrm{pg} / \mathrm{mL}(<46 \mathrm{pg} / \mathrm{mL}$ ), dehydroepiandrosterone sulfate (DHEA-SO4) of $699 \mu \mathrm{g} / \mathrm{dL}(80-560 \mu \mathrm{g} / \mathrm{dL}$ ), testosterone of $167 \mathrm{ng} / \mathrm{dL}$ (241-827 $\mathrm{ng} / \mathrm{dL})$, estradiol of $83.1 \mathrm{pmol} / \mathrm{L}(<146.1 \mathrm{pmol} / \mathrm{L})$ and normal urinary metanephrines. A renal CT scan confirmed a right adrenal mass of $49 \times 64 \times 56 \mathrm{~mm}$ (Figure 1).

The patient was evaluated by the general surgery department and he underwent 18F-fluorodeoxyglucose positron emission tomography (FDG-PET) and magnetic resonance imaging (MRI) for better assessment of the nature and limits of the lesion. The FDG-PET (Figure 2) 


\section{Figure 1: Renal computed tomography scan}

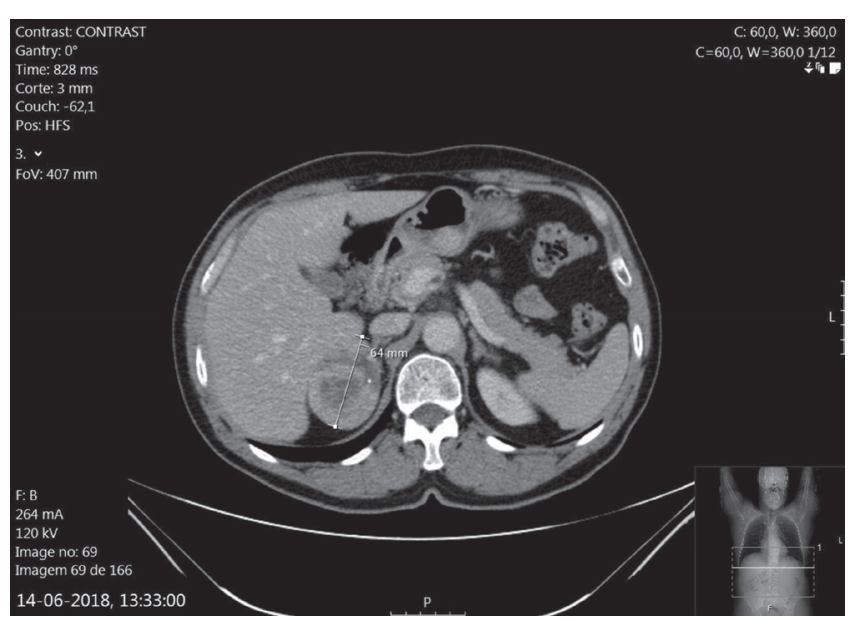

Heterogeneous nodular formation in the right adrenal gland, with enhanced areas after intravenous contrast and with a little calcification, not compatible with adenoma; the hypothesis of neoplasia has to be considered.

Figure 2: 18F-fluorodeoxyglucose positron emission tomography

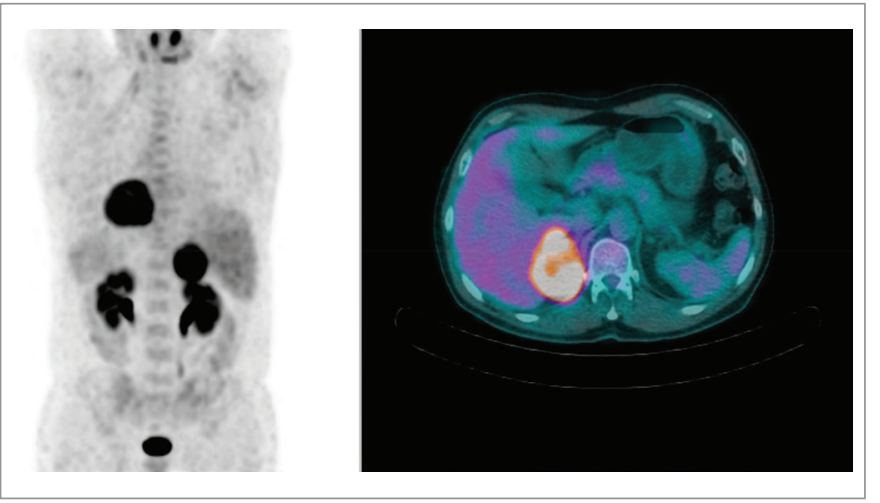

Bright and heterogeneous enhancement in the right adrenal mass, with greater expression in its posterior and inferior faces (SUVmax:16.4). In conclusion,

hypermetabolic lesion in the right adrenal gland, probably of malignant aetiology.

\section{Figure 3: Magnetic resonance imaging}

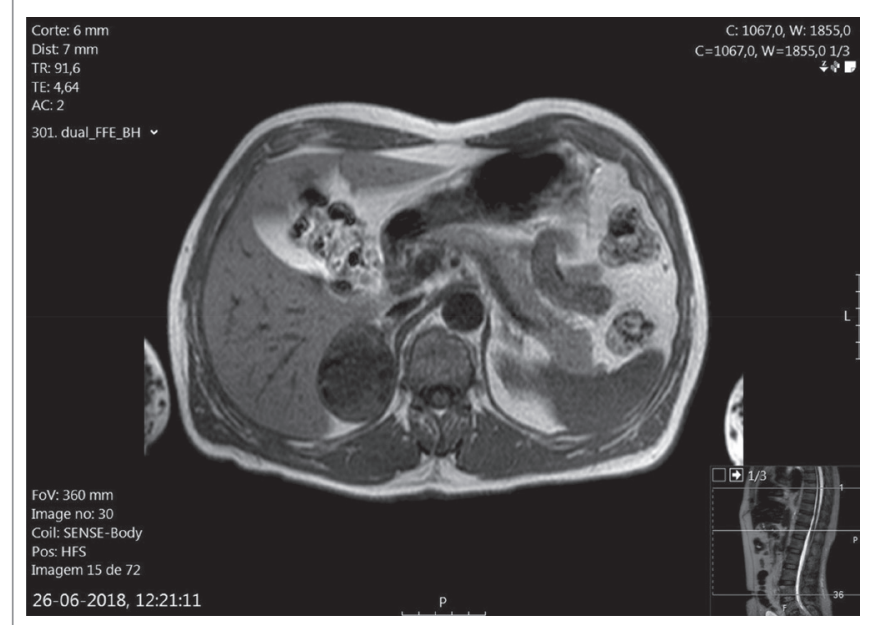

Nodule of $6.6 \times 4.7 \times 5.2 \mathrm{~cm}$ in the right adrenal gland, with heterogeneous texture, T2-hypersignal and T1-hyposignal, microscopic fat and areas of necrosis, which suggest primary carcinoma of the adrenal gland. showed an 18F-FDG hypermetabolism in the right adrenal mass (SUVmax: 16.4) and the MRI (Figure 3) detected a nodule of $66 \times 47 \times 52 \mathrm{~mm}$ in the right adrenal gland, without invasion signs.

In August 2018 the patient underwent right adrenalectomy by laparotomy with the collaboration of the adrenal and hepatobiliary surgical teams. To prevent postoperative adrenal insufficiency, the patient was medicated with hydrocortisone. He recovered uneventfully. The operative piece weighed $124 \mathrm{~g}$, measured $9.5 \times 5.5 \times 5.0 \mathrm{~cm}$, with a nodule of $6.0 \times 5.5 \times 5.0 \mathrm{~cm}$ of well-defined limits, spongy, brownish, with haemorrhage areas. The histological analysis (Figures 4 and 5) revealed the diagnosis of adrenocortical neoplasm with adenoma features with predominance of oncocytic cells (adrenocortical oncocytoma).

One month after surgery, the patient was asymptomatic, without signs of a surgical scar infection or incisional hernia. Four months after adrenalectomy, the general surgery team scheduled the next evaluation at 1-year post-operation, due to the patient's positive recovery. In February 2019, the patient was evaluated in the endocrinology department and displayed normalised analytical parameters: cortisol $13.1 \mu \mathrm{g} / \mathrm{dL}$ (4.3-22.4 $\mu \mathrm{g} / \mathrm{dL}$ ) and DHEA-SO4 $120 \mu \mathrm{g} / \mathrm{dL}$ (80-560 $\mu \mathrm{g} / \mathrm{dL})$. He is currently in follow up.

\section{Discussion}

Oncocytic cells were first described in 1931 by H Hamperl as cells with eosinophilic and granular cytoplasm.9 Recent advances have allowed us to understand that these features were a result of numerous mitochondria. ${ }^{9}$ Oncocytic tumours arise frequently from the kidneys, representing $3-7 \%$ of all renal neoplasms, ${ }^{7}$ but are already found in thyroid, pituitary gland, salivary glands, parathyroid glands, respiratory and gastrointestinal tracts. ${ }^{2-4}$ Adrenal oncocytomas are extremely rare, with nearly 200 published cases since Kakimoto et al. reported the first case in 1986. 5,6 The mechanisms for oncocytosis are not well understood and there are two theories: the first defends that mitochondria proliferation is a result of a genetic mutation and the second that it is a consequence of an epigenetic event resulting from cellular hypoxia. 10,11

Adrenal oncocytoma can occur in a large age range (15-77 years), more frequently in women (2.5:1.0), in the left adrenal gland (3.5:1.0), without known risk factors. 7,12 Frequently, an adrenal oncocytic neoplasm is discovered incidentally and is a rare entity to consider in the differential diagnosis of an adrenal incidentaloma. Differential diagnoses include cortical adenoma, pheochromocytoma, Cushing's syndrome, adrenocortical carcinoma, granulomas, adrenal cyst, myelolipoma, ganglioneuroma and metastatic disease.? Although it is, by definition, non-functioning, recent data indicate that $17.0-31.5 \%$ of adrenal oncocytomas have hormone production..$^{12}$ In our case report, $1 \mathrm{mg}$ overnight dexamethasone suppression test revealed probable autonomous cortisol secretion. Adrenal oncocytoma has been diagnosed in the context of Cushing's Syndrome, pheochromocytoma, aldosteronoma, pseudoprecocious puberty and virilising symptoms in females. ${ }^{78,12}$ The exclusion of pheochromocytoma is particularly important in the diagnosis of adrenal oncocytoma as it affects pre- and post-operative procedures.?

Imaging methods (e.g. CT, MRI) lack diagnostic capacity for adrenal oncocytoma as it is difficult to differentiate it from a malignant lesion.7.8 Imaging features of adrenal oncocytoma include a size of generally $>6 \mathrm{~cm}$, lipid-poor, a higher attenuation on $\mathrm{CT}$ ranging from 20-40 $\mathrm{HU}$, and malignant process features such as fibrous encapsulation or heterogeneous contrast enhancement? According 
Figure 4: Histological analysis (haematoxylin/eosin stain, 100x amplification) identifying neoplasm interface with normal gland

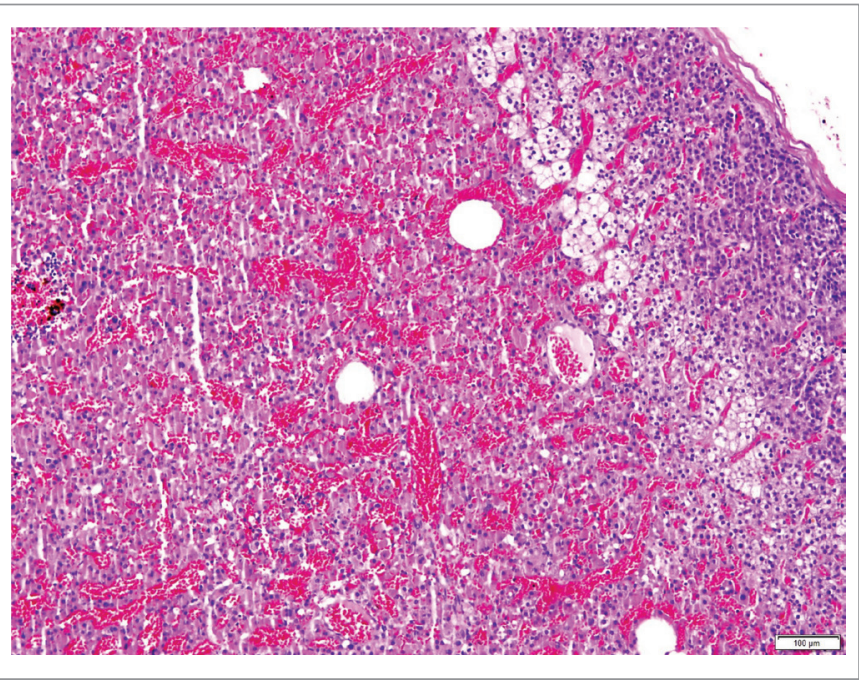

to the latest guidelines, an adrenal incidentaloma of $>4 \mathrm{~cm}$ and with more than $10 \mathrm{HU}$ is likely malignant and surgery must be considered. ${ }^{13}$ Despite this fact, an adrenal oncocytoma is typically benign. ${ }^{7}$ Recent studies report a $22 \%$ incidence of malignant oncocytomas. ${ }^{7.12}$ The diagnosis depends on the pathological and histological examination of the operative piece. Macroscopically, adrenal oncocytoma appears round, encapsulated, with an average diameter of $8 \mathrm{~cm}$, dark brown in colour with haemorrhage and necrosis areas. Histologically it is mainly composed of oncocytic cells. ${ }^{7.8}$

The treatment of choice for any suspicious adrenal mass is adrenalectomy, which may be by laparotomy when handling big lesions, or by laparoscopy for smaller lesions and if imaging displays a well-encapsulated tumour without invasion?

Prognosis is determined by the characteristics of the oncocytoma. Adrenocortical tumours can be defined using the Weiss criteria of malignancy ${ }_{1}^{14}$ which was adapted by Bisceglia et al. in order to distinguish malignant oncocytic tumours. ${ }^{15}$ The Lin-Weiss-Bisceglia risk system includes: major criteria (mitotic rate of $>5$ mitoses/50 high power fields, atypical mitosis and venous invasion), minor criteria (>10 cm of diameter and/or >200 g of weight, necrosis, capsular or sinusoidal invasion) and definitional criteria (dominance of oncocytic cells, high nuclear grade and diffuse architectural pattern). The presence of one major criterion classifies the oncocytoma as carcinoma; the presence of one or more minor criteria classifies it as having uncertain malignant potential; and the absence of criteria indicates benign behaviour. ${ }^{8,15}$

Follow-up recommendations are scarce; on one hand there is no record of recurrence of benign or indeterminate potential oncocytomas after surgery; however, malignant oncocytomas have a 5-year survival rate of $50-60 \%$ after surgery. ${ }^{7.8}$ of nine malignant cases, five were declared disease-free at 6 months, while four exhibited recurrence.
Figure 5: Histological analysis (haematoxylin/eosin stain, 400x amplification) revealing a neoplasm consisting of cells with abundant eosinophilic cytoplasm

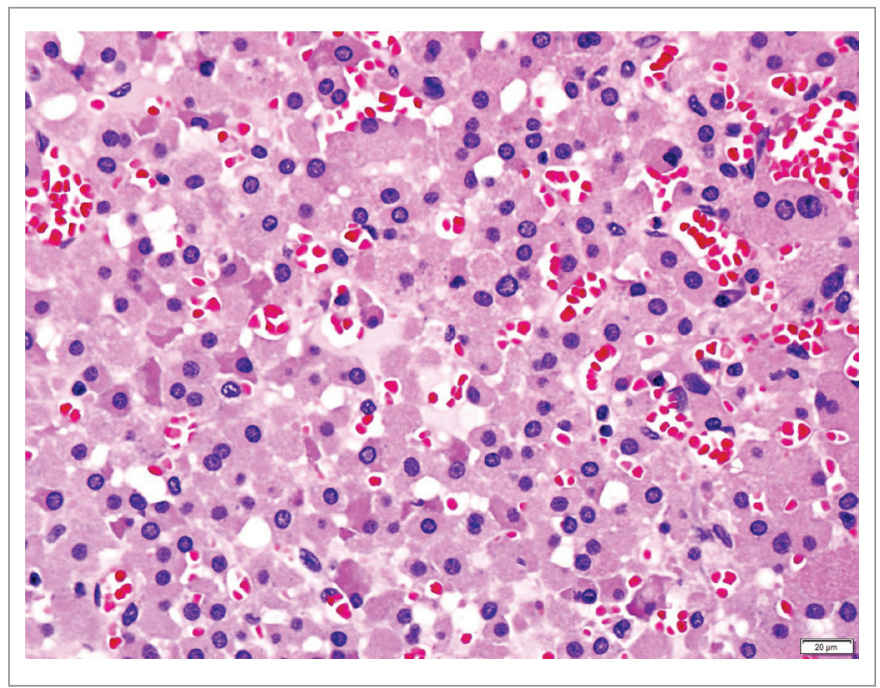

It is therefore advisable, in the absence of evidence, to maintain a regular follow up for a minimum of 5 years. ${ }^{7.8}$

\section{Conclusion}

The case of a 55-year-old male who presented with abdominal pain has been described here. Abdominal ultrasound revealed an adrenal heterogeneous lesion that measured $49 \times 64 \times 56 \mathrm{~mm}$ on CT scan. The patient was referred for right adrenalectomy by laparotomy under the suspicion of a carcinoma. Histological analysis revealed an adrenocortical oncocytoma. One-month post-surgery, the patient had recovered well and was asymptomatic.

A large $(>4 \mathrm{~cm})$, heterogeneous, lipid-poor ( $>10 \mathrm{HU}$ ) adrenal incidentaloma with heterogeneous contrast enhancement and necrosis areas is suggestive of adrenocortical carcinoma and surgery is mandatory. Plasma or urinary metanephrines are of particular importance as a pheochromocytoma diagnosis can affect pre-operative and postoperative management; however, a $1 \mathrm{mg}$ overnight dexamethasone suppression test, sex hormone levels, steroid precursors and aldosterone/renin ratio in the presence of hypertension or unexplained hypokalaemia are recommended.

Since imagiological characteristics of an oncocytoma are indissociable from the ones of a carcinoma, the diagnosis is determined through histological analysis. It is by definition a benign lesion, although it can be classified as having uncertain malignant potential or as carcinoma, with important prognosis and follow-up implications.

Adrenal oncocytoma is extremely rare, with less than 200 published cases, which makes it difficult to study, determine prognosis and define follow-up strategies. This case is a contribution to the knowledge on adrenal oncocytomas, since there is scarce information based on singular experiences. $\square$
1. Shah VN, Premkumar A, Walia R, et al. Large but benign adrenal mass: adrenal oncocytoma. Indian J Endocrinol Metab. 2012:16:469-71.

2. Maximo V, Rios E, Sobrinho-Simoes M. Oncocytic lesions of the thyroid, kidney, salivary glands, adrenal cortex, and parathyroid glands. Int I Surg Pathol. 2014;22:33-6
3. Tsuta K, Kalhor N, Raso MG, et al. Oncocytic neuroendocrine tumors of the lung: histopathologic spectrum and immunohistochemical analysis of 15 cases. Hum Pathol. 2011:42:578-85.
4. Kozakiewicz J, Teodorowicz E, Haczyńska-Partyka A, et al. Adenoma oxyphillicum, an extremely rare case of tumour of the larynx end cancer lungs which are going together.

Otolaryngol Pol. 2007;61:311-4

Kakimoto S, Yushita Y, Sanefuji T, et al. Non-hormonal adrenocortical adenoma with oncocytoma-like appearances Hinyokika Kiyo. 1986;32:757-63.

Dong D, Liu X, Ji Z, Li H. Diagnosis and treatment of adrenocortical oncocytoma: case report of five cases and review of the literature. Front Oncol. 2019:9:338. 
7. Botsios D, Blouhos K, Vasiliadis K, et al. D. Adrenocortical oncocytoma - a rare tumor of undefined malignant potential: report of a case. Surg Today. 2007;37:612-7.

8. Peacock J, English L, Mahesan T, et al. Adrenal oncocytoma: Peacock J, English L, Mahesan T, etal. Ad case report and review of the 9:2:33-7

. Mete O, Asa SL. Aldosterone-producing adrenal cortica adenoma with oncocytic change and cytoplasmic eosinophilic globular inclusions. Endocr Pathol. 2009;20:182-5.
10. Hoang MP, Ayala AG, Albores-Saavedra J. Oncocytic adrenocortical carcinoma: a morphologic,

immunohistochemical and ultrastructural study of four cases. Mod Pathol. 2002;15:973-8

11. Yazhao $\mathrm{H}$, Yuanyuan $\mathrm{H}$, Jinghai $\mathrm{H}$, et al. Adrenocortical oncocytoma 11 case reports and review of the literature. Medicine 2017:96:(e8750).

12. Mearini L, Del Sordo R, Costantini E, et al. Adrenal oncocytic neoplasm: a systematic review. Urol Int. 2013:91:125-33.

13. Fassnacht M, Arlt W, Bancos I, et al. Management of adrena incidentalomas: European Society of Endocrinology Clinica Practice Guideline in collaboration with the European Network for the Study of Adrenal Tumors. Eur J Endocrinol. 2016:175:G1-G34.

14. Medeiros $\sqcup$, Weiss $L M$, New developments in the pathologic diagnosis of adrenal cortical neoplasms. A review. Am J Clin Pathol. 1992;97:73-83.

15. Bisceglia M, Ludovico O, Di Mattia A, et al. Adrenocortical oncocytic tumors: report of ten cases and review of the literature. Am I Surg Pathol. 2004;12:231-3. 